\title{
Effect of Sesamin on Apoptosis and Cell Cycle Arrest in Human Breast Cancer MCF-7 Cells
}

\author{
An-Ci Siao ${ }^{1 \&}$, Chien-Wei Hou ${ }^{2 \&}$, Yung-Hsi Kao ${ }^{1}$, Kee-Ching Jeng ${ }^{3 *}$
}

\begin{abstract}
Dietary prevention has been known to reduce breast cancer risk. Sesamin is one of the major components in sesame seeds and has been widely studied and proven to have anti-proliferation and anti-angiogenic effects on cancer cells. In this study, the influence of sesamin was tested in the human breast cancer MCF-7 cell line for cell viability (MTT assay) and cell cycling (flow cytometry). Results showed that sesamin dose-dependently (1, 10 and $50 \mu M$ ) reduced the cell viability and increased LDH release and apoptosis (TUNEL assay). In addition, there was a significant increase of sub-G1 phase arrest in the cell cycle after sesamin treatment. Furthermore, sesamin increased the expression of apoptotic markers of Bax, caspase-3, and cell cycle control proteins, p53 and checkpoint kinase 2 . Taken together, these results suggested that sesamin might be used as a dietary supplement for prevention of breast cancer by modulating apoptotic signal pathways and inhibiting tumor cell growth.
\end{abstract}

Keywords: Sesamin - breast cancer - apoptosis - cell cycle

Asian Pac J Cancer Prev, 16 (9), 3779-3783

\section{Introduction}

Breast cancer is one of the most common cancers among women, and is the leading cause of death globally (Jemal et al., 2011). Many women still die of this disease in spite of cancer therapy, therefore new treatment strategies are needed. Natural dietary substances have been used for reducing the incidence of cancer (Gullett et al., 2010) and natural compounds can downregulate anti-apoptotic proteins (Kong et al., 2014). Cancer cells are also known to lose the ability to negatively regulate the cell cycle leading to their continuous proliferation. The cell cycle is controlled by numerous mechanisms ensuring correct cell division including regulation of cyclin-dependent kinases (CDK) by cyclins, CDK inhibitors and phosphorylating events (Caldon et al., 2010). Cancer cells activate the apical kinase ATM (Ataxia Telangiectasia Mutated) and its target and effector checkpoint kinase Chk2 after DNA damage by genotoxic substance (Ciccia and Elledge, 2010). Following DNA damage, ATM phosphorylates Chk2 on Thr68, leading to its activation and phosphorylation of several substrates, including p53, HDMX, PML, TRF2 (Ouchi and Ouchi, 2014). A wide range of phytochemicals derived from medicinal plants have been reported to exhibit anticancer activity by targeting the cell cycle regulation and apoptosis. Sesamin, a major lignin from sesame seeds has been reported to prevent hypertension, protect brain and liver injuries (Noguchi et al., 2001; Hsieh et al., 2011; Chiang et al., 2014) and modulate the cell cycle in human cancer cells (Yokota et al., 2007).
We report here the effect of sesamin on the cell cycle and apoptosis of MCF-7 breast cancer cells

\section{Materials and Methods}

\section{Reagents}

Fetal bovine serum (FBS) was obtained from Gibco Invitrogen (Grand Island, NY, USA). Dulbecco's Modified Eagle's medium (DMEM) were purchased from GIBCO (Grand Island, NY, USA). Sesamin was kindly provided from Joben Bio-Medical Co. (Kaohsiung, Taiwan). Antibodies were obtained from various sources: $\beta$-Actin (mouse, 1:5000; Novus, Littleton, CO, USA), caspase-3 (rabbit, 1:1000; Cell Signaling, Danvers, MA, USA), p21WAF1 (mouse, 1:5000; Cell Signaling), p53 (mouse, 1:2000, Calbiochem, Darmstadt, Germany), human phospho-Chk2 ELISA kit (R and D, Minneapolis, MN, USA), Bax (mouse, 1:5000; Cell Signaling), and anti-rabbit or anti-mouse IgG $(\mathrm{H}+\mathrm{L})$ (Jackson ImmunoResearch, West Grove, PA, USA).

\section{Cell lines and culture}

Human breast cancer MCF-7 cell line was purchased from ATCC. Cells were cultured in a humidified incubator at $37^{\circ} \mathrm{C}$ and $5 \% \mathrm{CO}_{2}$ atmosphere in DMEM (Dulbecco's modified Eagle's medium), supplemented with $10 \%$ FBS and $1 \%$ penicillin-streptomycin.

\section{Cell viability assay}

Cell viability was measured using blue formazan

${ }^{1}$ Department of Life Sciences, National Central University, Jhongli, ${ }^{2}$ Department of Biotechnology and Pharmaceutical Technology, Yuanpei University, Hsinchu, ${ }^{3}$ Department of Pharmacy, Tungs' Taichung MetroHarbor Hospital, Taichung, Taiwan ${ }^{\circledR}$ Equal contributors*For correspondence: kcjeng@gmail.com 
An-Ci Siao et al

that was metabolized from colorless 3-(4,5-dimethylthiazol-2-yl)-2,5-diphenyl tetrazolium bromide (MTT) by mitochondrial dehydrogenases, which are active only in live cells. MCF-7 cells were preincubated in 24-well plates at a density of $5 \times 10^{5}$ cells per well for $8 \mathrm{~h}$ and then treated with various concentrations of sesamin $(1,10$, and $50 \mu \mathrm{M}$ ) for $24 \mathrm{~h}$. The cells were washed by $1 \times$ PBS solution, and then incubated in $0.5 \mathrm{mg} / \mathrm{ml} \mathrm{MTT}$ at $37^{\circ} \mathrm{C}$ for $1 \mathrm{~h}$. A solubilization solution, $200 \mu \mathrm{l}$ of DMSO (dimethyl sulfoxide), was added to each well and absorption values read at $540 \mathrm{~nm}$ on microtiter plate reader (spectraMAX 340, Molecular Devices, Sunnyvale, CA, USA). Data were expressed as the mean percent of viable cells vs. control.

\section{LDH assay}

Cytotoxicity was assessed by meaning the release of the cytosolic enzyme lactic dehydrogenase (LDH) from damaged cells. LDH released from the $24 \mathrm{~h} \mathrm{MCF-7} \mathrm{cell}$ culture was using an LDH diagnostic kit (Boehringer Mannheim, Mannheim, Germany). Absorbance values were recorded at 490 and $630 \mathrm{~nm}$ with the aid of a SpectraMAX340 reader.

\section{Cell cycle analysis}

The effect of sesamin on cell cycle distribution was assessed by flow cytometry after staining the MCF-7 cells with propidium iodide $(\mathrm{PI})$. Briefly, the cells $\left(1 \times 10^{5} / \mathrm{ml}\right)$ were treated with different sesamin concentrations $(1,10$ and $50 \mu \mathrm{g} / \mathrm{ml}$ ) for $48 \mathrm{~h}$. The treated cells were harvested, washed with PBS and fixed with $70 \%$ ethanol on ice. Then cells washed with cold PBS, suspended in $200 \mu \mathrm{L} 1 \mathrm{X}$ Propidium Iodide + RNase staining solution and incubate at $37^{\circ} \mathrm{C}$ in the dark for $30 \mathrm{~min}$. Propidium Iodide Flow Cytometry Kit was used for cell cycle analysis. DNA content of the cells was measured by C6 flow cytometer and the population of each phase was determined using CFlow Plus analysis software (BD Accuri Cytometers, Ann Arbor, MI, USA).

\section{TUNEL assay}

The TUNEL assay uses an enzyme (terminal deoxynucleotidyl transferase) to add biotinylated nucleotides to the strand breaks found in the DNA of apoptotic cells. TUNEL assay was performed by the manufacture's protocol. Briefly, sesamin treated, or nontreated MCF-7 cells were cultured in 6-well plates for $24 \mathrm{~h}$. After the incubation period, the culture medium was aspirated, and the cell layers were trypsinized. The trypsinized cells were reattached on $0.01 \%$ polylysinecoated slides, fixed with $4 \%$ methanol-free formaldehyde solution, and stained according to the TUNEL system protocol (BioVision, Milpitas, CA, USA). The stained cells were observed using a fluorescence microscope with excitation at 450-500 $\mathrm{nm}$ and detection wavelength at 515$565 \mathrm{~nm}$. To determine the percentage of cells undergoing apoptosis, 100 cells were counted in each experiment.

\section{CHK2 assay}

Cell lysate samples were centrifuged (2000 x g for 5 $\mathrm{min})$ to pellet cells and cell debris. The supernatants were analyzed using an ELISA for Chk2 phosphorylated at threonine 68 by human phospho-Chk2 (T68) kit (R and D) and measured at $450 \mathrm{~nm}$ with an ELISA reader according to the manufacturer's instructions.

\section{Western blotting}

Protein samples from sesamin-treated or untreated cells, containing $40 \mu \mathrm{g}$ of protein each, were separated on $11.5 \%(\mathrm{w} / \mathrm{v})$ sodium dodecyl sulfatepolyacrylamide gels and transferred to Immobilon polyvinylidene difluoride membranes (Millipore, Bedford, MA, USA). The membranes were incubated with rabbit anti-mouse beta-actin (Rockland, Gilbertsville, PA, USA), caspase-3, or anti-phospho-MAPKs (Cell Signaling, Danvers, MA, USA). The membranes were subsequently incubated with conjugated goat anti-rabbit IgG (PerkinElmer, Boston, MA, USA). p53, p21, Bax, and caspase-3 proteins were detected by chemiluminescence detection system (Western Lightning ${ }^{\circledR}$ Plus-ECL, PerkinElmer, Waltham, MA, USA) and quantified with a densitometric scanner (PDI, Huntington Station, NY, USA).

\section{Statistics}

All data are expressed as mean \pm S.D. For single variable comparisons, Student's t-test was used. For multiple variable comparisons, data were analyzed by one-way ANOVA using by Dunnett's test. P values of less than 0.05 or 0.01 were considered significant.

\section{Results}

Cytotoxic effect of sesamin on the viability of MCF-7 cells

Sesamin was tested for cytotoxic effect on the MCF-7 cells. Sesamin (10 and $50 \mu \mathrm{M})$ decreased the cell viability by 18 and $30 \%$ and increased LDH release, 22 to $50 \%$,

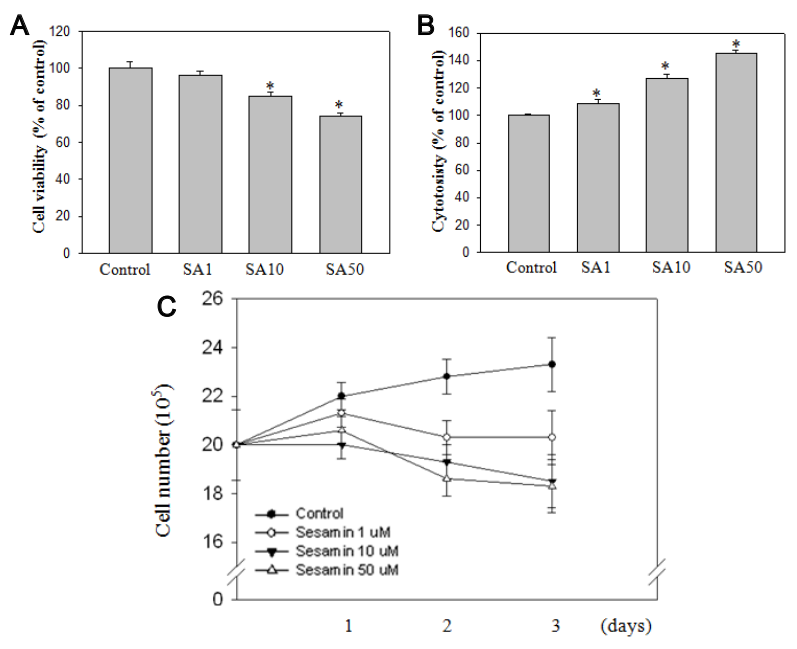

Figure 1. The Effect of Sesamin on the Viability and Cytotoxicity on MCF-7 Cells. The cells were treated with sesamin $(0,1,5,10 \mu \mathrm{M})$ for $24 \mathrm{~h}$. The cell viability $\mathbf{A})$ and cytotoxicity B) was determined by MTT and LDH assay, respectively. Data are expressed as the mean \pm SD of three separate experiments. ${ }^{*} P<0.01$ as compared to the control. C) Proliferation of MCF-7 cells. The cells grown on $20 \mathrm{~cm}^{2}$ culture dish were treated with sesamin $(0,1,5,10 \mu \mathrm{M})$ for 24 to $72 \mathrm{~h}$ and separated by trypsin EDTA. Viable cells were enumerated by trypan blue exclusion with an aid of hemocytometer. $* P<0.01$ as compared to the control 
respectively (Figure 1a, b). This effect was consistent with the number of viable cells from three days culture (Figure 1c). Sub-G1 phase arrest was increased 19,27, and $41 \%$, respectively by sesamin treatment $(1,10$, and $50 \mu \mathrm{M})$ as compared to $16 \%$ of the control (Figure 2). Cell apoptosis was also increased by sesamin dose-dependently from TUNEL assay $(P<0.01$, Figure 3$)$.
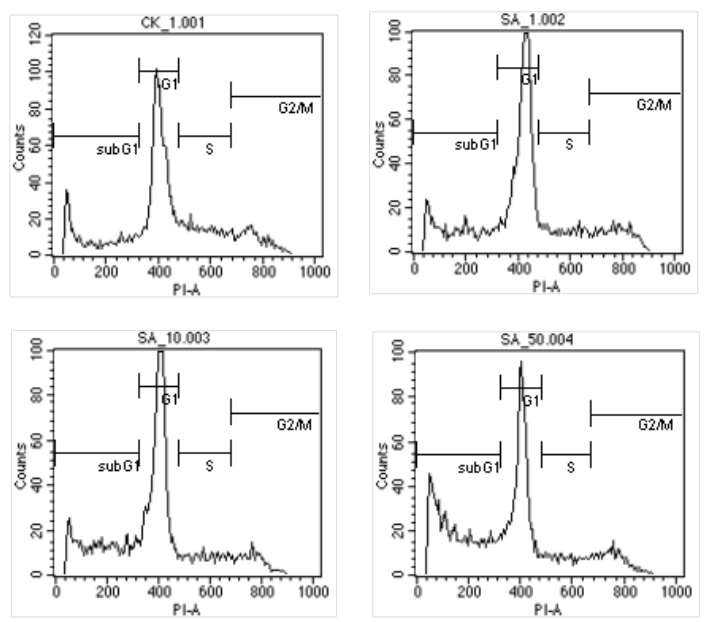

Figure 2. The Effect of Sesamin on Cell Cycle of MCF7 Cells. The cells grown on $20 \mathrm{~cm}^{2}$ culture dish were exposed to sesamin $(0,1,5,10 \mu \mathrm{M})$ for $24 \mathrm{~h}$. The cells were fixed with $70 \%$ ethyl alcohol and then stained with propidium iodide. The cell cycle was analyzed by flow cytometer
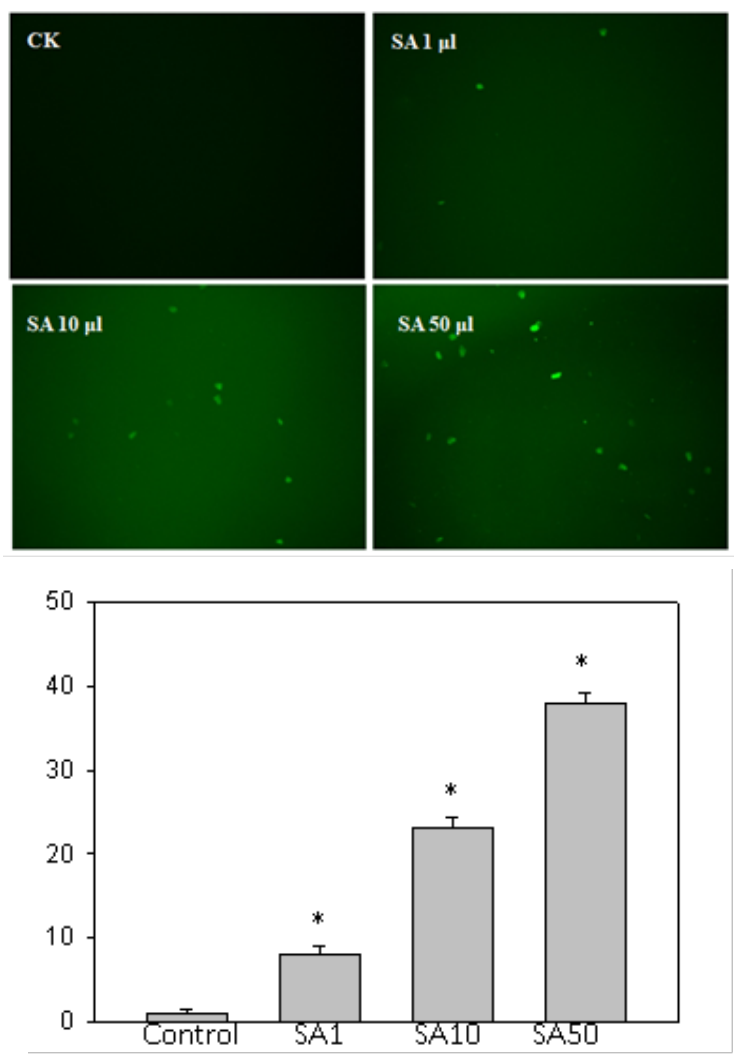

Figure 3. The Effect of Sesamin on the Apoptosis of MCF-7 Cells. Cell apoptosis was determined with TUNEL assay after $24 \mathrm{~h}$ of exposure to sesamin $(0,1,5,10 \mu \mathrm{M})$. Values represent the mean from three independent experiments. ${ }^{*} p<0.05$ $v s$. the control

$$
\text { Control SA.1 SA.10 SA.50 ( } \mu \mathrm{M})
$$
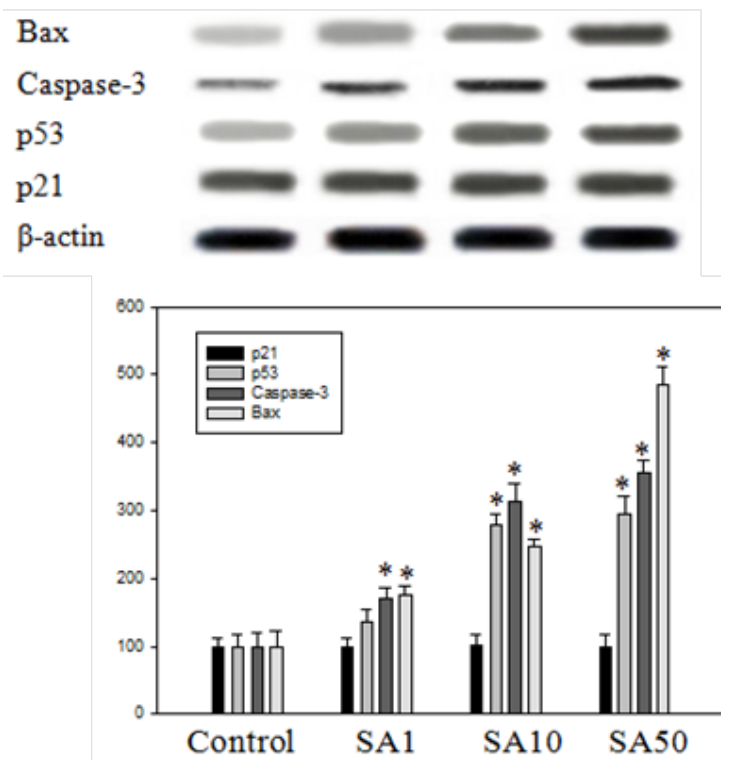

Figure 4. Effect of Sesamin Treatment on BAX, Caspase-3, p53 and p21 expression of MCF-7 Cells. Western blots results of scanning densitometer analysis are presented as value of proteins/beta-Actin. Values represent the mean from three independent experiments (lower panel). $* P<0.05$, vs. non-treated control

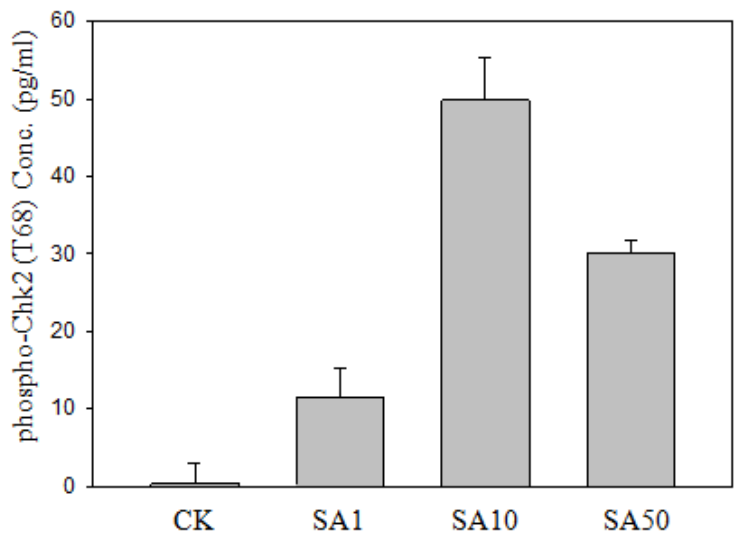

Figure 5. Effect of sesamin treatment on Chk2 expression of MCF-7 cells. Phosphorylated Chk2 in MCF-7 cells was increased significantly by sesamin as compared to that of the control $(P<0.01)$

\section{Cell cycle and apoptotic signaling pathways}

Key cell cycle control proteins p53 and p21 and apoptotic proteins caspase- 3 and Bax were examined by western blots. The results showed that MCF-7 cells treated with sesamin (1-50 $\mu \mathrm{M})$ for $10 \mathrm{~min}$ increased Bax (75 310\%), caspase-3 (70 187\%), and p53 (35 194\%) but not p21 protein expression ( $0 \%$, Figure 4$)$. Cell cycle checkpoints for damaged or abnormally structured DNA can slow or arrest the progression of cell-cycle (Ouchi and Ouchi, 2014). The result showed an increased phosphory Chk2 in MCF-7 cells by sesamin as compared to that of the control $(p<0.01$, Figure 5). 


\section{Discussion}

In the present results, sesamin reduced the cell viability by inducing both necrosis and apoptosis in MCF-7 cells, as shown by LDH release and TUNEL assay, respectively. This result agreed with a study in the nude mice supplemented with $1 \mathrm{~g} / \mathrm{kg}$ sesame seed reduced MCF-7 tumor size by $23 \%$ compared to control (Truan et al., 2012).

Several components of the apoptotic pathways were examined for the reduced cell viability by sesamin. The proteins of the Bcl-2 family play an essential role in apoptosis that BAX increases during apoptosis (Hasan et al., 2011). Our result showed that an increase of BAX expression in MCF-7 cells by sesamin. Furthermore, activated caspase- 3 that played a crucial role in the final step of apoptosis (Vegran et al., 2011) was increased by sesamin. These data were consistent with results of natural compounds through p53/caspase-3 induced apoptosis of MCF-7 cells (Esmaeili-Mahani et al., 2014). Our result also demonstrated a sub-G1 arrest in the cell cycle by sesamin. The cell cycle arrest was related with the increased expression of tumor suppressor p53. p53 plays an important role in response to DNA damage or other genomic instability. Functional p53 protein is crucial in p53-dependent pathway leading to cell cycle arrest or apoptosis (Lee et al., 2014). Several stresses lead to the activation of p53 and results in the arrest of cell cycle by increasing p 21 or by inhibiting cyclins and CDKs directly (Yadav et al., 2012; Kim et al., 2015). The involvement of p53 and its downstream CDK inhibitor p21 in sesamin-treated MCF-7 cells was then investigated. However, present result showed that p53 but not p21 protein level was increased significantly with increasing dose of sesamin. These results indicate that a p53 was partly involved sub-G1 arrest by sesamin in the cell-cycle of MCF-7 cells.

We further examined the effect of sesamin on phosphorylation of checkpoint $\mathrm{Chk} 2$ and found that phosphorylation of Chk2 was increased by sesamin and suggested that would allow the accumulation of sub-G1 phase in the cell cycle. This is different from a study that reports an arrest at the G1 phase by sesamin with down-regulated cyclin D1 expression (Yokota et al., 2007). However, treatment to lung NSCLC cancer cells with obtusilactone A and (-)-sesamin from Cinnamomum kotoense induce sub-G1 arrest from the DNA damage responses, including $\mathrm{G} 1 / \mathrm{S}$ checkpoint activation and apoptosis by phosphorylation of checkpoint proteins (H2AX, Nbs1, and Chk2), caspase-3 cleavage (Wang et al., 2010). Ellagic acid from pomegranate, muscadine grapes, walnuts and strawberries, has been shown to induce apoptosis and arresting cell cycle in the G0/G1 phase (Chen et al., 2015). In addition, rapamycin, a cytotoxic drug, shows characteristic features of apoptosis and an arrest in the G0/G1 phase to MCF-7 cells (Tengku Din et al., 2014). Therefore, increased phosphorylated Chk-2 by sesamin could lead to the Go/G1 arrest.

In conclusion, we demonstrate that anticancer activity of sesamin to MCF-7 cells. It inhibits cell proliferation by inducing cell cycle arrest and apoptosis. This induction of cell cycle arrest is through the increasing of p53 and Chk2 and apoptosis is through the activation of the Bax and caspase-3 pathways. Future work will focus on isolation and characterization of the tentatively identified major compound and will explore the prospective in vitro and in vivo anticancer capabilities.

\section{Acknowledgements}

This work was supported by a grant TTMHH103R0004 from Tung's Taichung MetroHarbor Hospital.

\section{References}

Caldon CE, Sutherland RL, Musgrove E (2010). Cell cycle proteins in epithelial cell differentiation: implications for breast cancer. Cell Cycle, 9, 1918-28.

Chen HS, Bai MH, Zhang T, Li GD, Liu M (2015). Ellagic acid induces cell cycle arrest and apoptosis through TGF- $\beta$ / Smad3 signaling pathway in human breast cancer MCF-7 cells. Int J Oncol, 46, 1730-8.

Chiang HM, Chang H, Yao PW, et al (2014). Sesamin reduces acute hepatic injury induced by lead coupled with lipopolysaccharide. J Chin Med Assoc, 77, 170-8.

Ciccia A, Elledge SJ (2010). The DNA damage response: making it safe to play with knives. Mol Cell, 40, 179-204.

Esmaeili-Mahani S, Falahi F, Yaghoobi MM (2014). Proapoptotic and antiprolifera- tive effects of Thymus caramanicus on human breast cancer cell line (MCF-7) and its interaction with anticancer drug vincristine. Evid Based Complement Alternat Med, 2014, 893247.

Gullett NP, Ruhul Amin AR, Bayraktar S, et al (2010). Cancer prevention with natural compounds. Semin Oncol, 37, 258-81.

Hsieh PF, CW Hou, PW Yao, et al (2011). Sesamin ameliorates oxidative stress and mortality in kainic acid-induced status epilepticus by inhibition of MAPK and COX-2 activation. J Neuroinflamm, $8,57$.

Jemal A, Bray F, Center MM, Ferlay J, Ward E, Forman D (2011). Global cancer statistics. CA Cancer J Clin, 61, 69-90.

Kim MY, Park SJ, Shim JW, et al (2015). Naphthazarin enhances ionizing radiation-induced cell cycle arrest and apoptosis in human breast cancer cells. Int J Oncol, 46, 1659-66.

Kong Y, Chen J, Zhou Z, et al (2014). Cucurbitacin E induces cell cycle $\mathrm{G} 2 / \mathrm{M}$ phase arrest and apoptosis in triple negative breast cancer. PLoS One, 9, 103760.

Noguchi T, Ikeda K, Sasaki Y, et al (2001). Effects of vitamin E and sesamin on hypertension and cerebral thrombogenesis in stroke-prone spontaneously hypertensive rats. Hypertens Res, 24, 735-42.

Ouchi M, Ouchi T (2014). Distinct DNA damage determines differential phosphorylation of Chk2. Cancer Biol Ther, 15, 1700-4.

Lee JO, Moon JW, Lee SK, et al (2014). Rhus verniciflua extract modulates survival of MCF-7 breast cancer cells through the modulation of AMPK-pathway. Biol Pharm Bull, 37, 794-801.

Tengku Din TA, Seeni A, Khairi WN, Shamsuddin S, Jaafar H (2014). Effects of rapamycin on cell apoptosis in MCF-7 human breast cancer cells. Asian Pac J Cancer Prev, 15, 10659-63.

Truan JS, Chen JM, Thompson LU (2012). Comparative effects of sesame seed lignan and flaxseed lignan in reducing the growth of human breast tumors (MCF-7) at high levels of circulating estrogen in athymic mice. Nutr Cancer, $\mathbf{6 4}$, 65-71. 
Végran F, Boidot R, Solary E, Lizard-Nacol S (2011). A short caspase-3 isoform inhibits chemotherapy-induced apoptosis by blocking apoptosome assembly. PLoS One, 6, 29058.

Wang HM, Cheng KC, Lin CJ, et al (2010). Obtusilactone A and (-)-sesamin induce apoptosis in human lung cancer cells by inhibiting mitochondrial Lon protease and activating DNA damage checkpoints. Cancer Sci, 101, 2612-20.

Yadav V, Sultana S, Yadav J, Saini N (2012). Gatifloxacin Induces $\mathrm{S}$ and $\mathrm{G} 2$-phase cell cycle arrest in pancreatic cancer cells via p21/p27/p53. PLoS One, 7, 47796.

Yokota T, Matsuzaki Y, Koyama M, et al (2007). Sesamin, a lignan of sesame, down-regulates cyclin D1 protein expression in human tumor cells. Cancer Sci, 98, 1447-53. 